\title{
Kandii Puzzle: Rompecabezas interactivo para apoyar la discapacidad intelectual
}

\author{
David May, Diana Arias, Lorena Baez, Miguel Angel \\ Rivera, Rafael Luna
}

Publicado: 31 Octubre 2018

\begin{abstract}
Resumen
Kandii Puzzle es una propuesta innovadora desarrollada mediante la metodología de Diseño Centrado en el Usuario Extendida (UCD-e). Consiste en la combinación de medios tradicionales (rompecabezas físico) con avances tecnológicos (como la proyección de imágenes) para crear herramientas interactivas que beneficiarán a los niños que poseen una discapacidad intelectual (DI) de nivel moderado, apoyándolos en el desarrollo de sus características sensoriales, motrices y cognitivas.
\end{abstract}

Palabras clave: Interacción Humano Computadora (HCI), Diseño Centrado en el Usuario extendido (UCD-e), Discapacidad intelectual, Retraso mental, Rompecabezas interactivo.

\section{Introducción}

En una escala nacional, los niños que presentan alguna discapacidad conforman el $8.8 \%$ del total de la población, de la cual el $26.6 \%$ muestran alguna discapacidad mental [6].

Existen cuatro niveles de DI: leve, moderado, intenso y profundo [1]. Este proyecto busca ayudar al desarrollo de las características sensoriales y motrices de los niños que presentan una DI de nivel moderado y poseen una edad mental de entre los 5 y 11 años, sin tomar en cuenta la edad cronológica.

\section{Problemática}

La DI afecta la forma de pensar, razonar y aprender de una persona. También la manera de cómo se comunica, se comporta y sus características sensoriales y motrices, alterando sus habilidades adaptativas, conceptuales (hablar y escribir), sociales (autoestima, responsabilidad, seguir normas) y prácticas (comer, vestirse) [4], cambiando sus necesidades y calidad de vida.

La intervención temprana favorecerá el desarrollo de sus características sensoriales (percepción, figura-fondo, sonidos reconocimiento de su cuerpo) y habilidades en la motricidad fina, así como a desarrollar habilidades lógicas [1].

\section{UCD Extendido}

Se utilizó la metodología UCD-e porque se centra en el

May, D., Arias, D., Baez, L., Rivera, MA., Luna, R.,

Universidad Tecnológica de la Mixteca

Heroica Ciudad de Huajuapan de León, Oaxaca

Email: davidmay@mixteco.utm.mx, dianaarias.19@hotmail.com,

lorena_bz_mtz@hotmail.com, river182@gmail.com,

rafael.luna.art@gmail.com "entendimiento", el cual influye durante todo el proceso [5]. El UCD-e permite obtener un sistema útil, implicando revisiones y evaluaciones constantes en cada proceso, además de mejorar la experiencia del usuario. Consta de cinco procesos fundamentales iterativos (Figura 1).

\subsection{Entendimiento}

Para comprender el tema de la DI se realizó una recopilación de información documentada, así como dos entrevistas a docentes especializados en la educación especial.

En una entrevista semi-estructurada realizada a la Profa. Olivia Ortiz Soriano, quien funge como educadora en el Centro de Atención Múltiple (CAM), ubicado en la H. Cd. de Huajuapan de León, Oaxaca, nos explicó que a los niños con este tipo de discapacidad se les facilita aprender por medio de la asociación y campos semánticos. También enfatizó que las habilidades más importantes que deben de desarrollar son la motricidad fina y la percepción figura-fondo.

Asimismo, en su entrevista la Profa. Mary Carmen Lara S., quien también funge como educadora en el CAM, destacó que los niños retienen información fácilmente por medio de técnicas de asociación. De igual forma comentó la importancia de enseñar el lenguaje y reconocimiento del cuerpo a los niños con discapacidad (en general), de manera que puedan identificar agresiones o abusos hacia ellos.

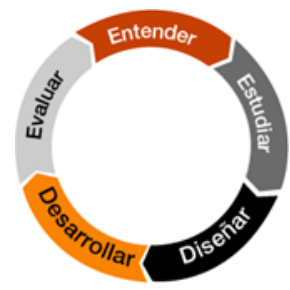

Figura 1. UCD-e

Según una revisión encargada por la Organización mundial de la Salud (OMS) indica que los niños con discapacidad son víctimas de violencia sexual con una frecuencia de 2,9 veces mayor que los no discapacitados. De igual manera, los niños cuya discapacidad incluye enfermedades mentales o intelectuales son aún más vulnerables, pues sufren violencia sexual con una frecuencia 4,6 veces mayor que sus homólogos sin discapacidad [7].

Un dato importante, mencionado en ambas entrevistas, es que los niños, con cualquier tipo de discapacidad, son muy curiosos 
con la tecnología. Por lo tanto, Kandii Puzzle pretende ser una herramienta tecnológica e innovadora que estimule el desarrollo de sus habilidades.

\subsection{Estudio}

Para realizar un estudio contextual, se contactó a un alumno del CAM, que cumplía con el perfil necesario: un niño o niña con un nivel socioeconómico bajo, con DI moderado, que presente limitaciones sensoriales y motrices de manera que requiera supervisión en cada actividad. Con una edad mental de entre los 5 y 11 años, etapa de la vida donde se intensifica el desarrollo intelectual, capacidades físicas, sociales y de identidad [3].

En un nivel de DI intenso o profundo se presenta la incapacidad de leer, calcular o entender lo que otros dicen [8], habilidades que son necesarias para completar las tareas de Kandii Puzzle, por lo tanto no es posible su uso en esos niveles de DI.

Mediante el estudio contextual se observó, con el consentimiento de los padres, el comportamiento del usuario en su domicilio (Figura 2). También se realizó una entrevista semiestructurada a los familiares a cargo, quienes compartieron su experiencia en el cuidado del usuario. Esto nos permitió conocer la vida cotidiana del usuario, sus habilidades y las que requiere reforzar, así como sus principales necesidades.

Los resultados de estas entrevistas se analizaron mediante la elaboración de reportes, de los cuales se comparó la información recabada de los familiares con la de los docentes, y junto con las observaciones, se identificaron las habilidades que se podrían reforzar mediante la estimulación temprana en la DI: la motricidad fina, la percepción y el lenguaje.

\subsection{Diseño}

Se realizó un análisis de los datos por todo el equipo, y mediante un Brainstorming se concluyó el uso de un rompecabezas. Esto debido a que el usuario tiene más acercamiento y accesibilidad a los juegos de mesa. Asimismo, se tomaron en cuenta los beneficios que el rompecabezas ofrece desde un inicio [9], como son:

- Desarrollo de la motricidad fina: en la coordinación de los movimientos musculares de los dedos.

- Habilidades cognitivas: estimuladas por las figuras y colores, mejorando la atención, memoria y resolución de problemas.

- Lógica: al establecer una estrategia de armado.

- Manejo de la frustración: el usuario en vez de frustrarse se debe concentrar en lograr su objetivo.

- Habilidades sociales: al armarlo en equipo.

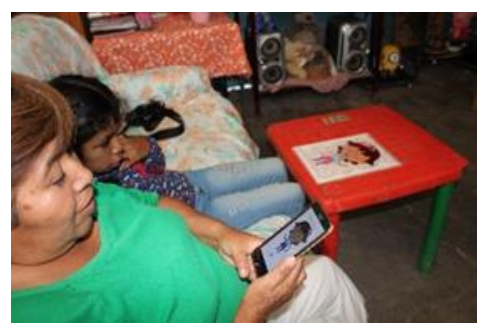

Figura 2. Usuarios en la evaluación

El implementar un rompecabezas interactivo implica que el usuario debe enfrentarse a conocer nuevos retos y resolverlos. De este modo, no sólo otorgará las ventajas ya mencionadas, sino que también ayudará al usuario a desarrollar sus características sensoriales, mediante sonidos y animaciones, además de que permitirá el acceso a diferentes temáticas lúdicas con una base de aprendizaje por asociación.
Es necesario mencionar que, con base a los datos de las entrevistas, es fundamental que el niño con DI sea guiado, por un tutor, durante el aprendizaje y desarrollo de sus habilidades. Para esto, se propone, como diseño conceptual, una aplicación móvil que controlará las funciones que ofrecerá el rompecabezas interactivo, permitiendo al tutor realizar explicaciones que favorezcan el desarrollo y aprendizaje del usuario.

\subsection{Construcción y funcionamiento}

\subsubsection{Rompecabezas: $1^{\circ}$ iteración}

Con la técnica de Brainstorming, se definió un rompecabezas que consistía en un mini proyector fijo sobre una mesa, proyectando una imagen sobre cada pieza del tablero. Cada pieza se identificaría con un código QR y de esta manera se sabría qué imagen se debe proyectar. Con ayuda de la técnica Paper Prototyping las ideas fueron ilustradas para una mayor comprensión, sin embargo, se encontró un inconveniente: si alguna parte del cuerpo del usuario entraba en el rango del proyector o si el usuario extraía la pieza fuera de este rango, ésta ya no tendría la imagen encima y sería difícil identificarla. También el código QR dificultaría la distinción de la imagen proyectada encima de la pieza.

\subsubsection{Rompecabezas: $2^{\circ}$ iteración}

Por medio de las técnicas de Paper Prototyping y Brainstorming se concluyó que el proyector debería tener una posición horizontal, de manera que ya no se interfiriera entre la proyección y las piezas, sin embargo se continuaba con el inconveniente de que, sacando la pieza del tablero se eliminaba la imagen.

\subsubsection{Rompecabezas: $3^{\circ}$ iteración}

Mediante Brainstorming se estableció que cada pieza pueda tener su propia pantalla LCD, no obstante, debido a la situación socioeconómica de nivel bajo, establecida en el perfil de usuario, esta opción dejó de ser factible. Por lo anterior no se llegó a técnicas de Paper Prototyping.

\subsubsection{Rompecabezas: $4^{\circ}$ iteración}

Finalmente en la cuarta reunión, con la técnica de Brainstorming, se consideró la tecnología de un proyector dentro de cada pieza, evitando el inconveniente al sacarlas del tablero y el costo. En esta propuesta se considera que en un futuro este tipo de tecnología será más accesible. Se continuó con el Paper Prototyping para entender la idea y quedar todos de acuerdo.

Prosiguiendo con el Mock-up, se estableció al acrílico como material para todo el rompecabezas debido a su resistencia, ligereza y superficie transparente, esto último permitiendo realizar la proyección de las animaciones. También se agregó una bocina para la asociación de sonidos.

Kandii Puzzle está constituido por 24 piezas, enfocado a crear asociaciones a partir de campos semánticos, donde el usuario puede detectar y conocer parte de su entorno y su contexto.

La ventaja principal es que las piezas pueden ser utilizadas para diferentes objetivos. Kandii Puzzle consta de 4 juegos diferentes, cada uno con sonidos y animaciones correspondientes:

- Cuerpo humano: El usuario puede aprender las partes principales de su cuerpo y diferenciar entre las públicas y privadas.

- Sílabas: Con las que el usuario podrá crear composiciones de palabras.

- Figura-Fondo: El usuario intentará percibir alguna figura escondida. 
- Familia: Para ayudar al reconocimiento de la familia, a través de la aplicación móvil se podrá tomar una foto familiar, para así ser proyectada sobre el rompecabezas.

\subsubsection{Aplicación móvil}

Durante la entrevista, la docente del CAM, comentó la necesidad de un tutor para guiar a un niño con DI en el correcto aprendizaje y estimulación de sus habilidades.

Por lo tanto, mediante Brainstorming, se decidió que una aplicación móvil permitiera un control total del rompecabezas de manera que facilite la interactividad, usabilidad y sobretodo, que se pueda seguir un ritmo de aprendizaje y estimulación, definido por el usuario del rompecabezas y controlado por el tutor mediante la aplicación móvil.

La interfaz para la aplicación móvil tiene un diseño minimalista para facilitar la usabilidad. El menú consta de 4 opciones, correspondientes a cada uno de los juegos ya descritos.

Cada juego tiene un menú para su configuración y un botón enfocado a mandar los datos al rompecabezas para que comience a funcionar.

\subsection{Evaluación}

Como ya se mencionó, Kandii Puzzle cuenta con cuatro juegos. En las evaluaciones se utilizó el juego del cuerpo humano con el objetivo de verificar que el diseño sea lúdico, usable y así ir mejorándolo conforme la experiencia de usuario. Las pruebas se realizaron con el mismo usuario, que participó en el estudio de contexto (Figura 2).

\subsubsection{Primera iteración}

- Rompecabezas. La primera prueba fue realizada mediante un rompecabezas no interactivo, de madera, con la finalidad de identificar las habilidades del usuario (alumno del CAM). Sus tareas fueron: 1) reconocer cada pieza y 2) armar el rompecabezas. La observación de usuario nos permitió analizar el contexto de uso, así como conocer las habilidades con las que cuenta el usuario. También se identificó que el usuario estaba familiarizado con los rompecabezas ya que concluyó su tarea en un tiempo considerablemente bajo.

- Aplicación móvil. Se presentó un prototipo de baja fidelidad al usuario (tutor) con la finalidad de identificar el nivel de usabilidad y si se encuentra familiarizado con la tecnología móvil. Nos percatamos que el usuario sí estaba familiarizado con esta tecnología, sin embargo presentó dificultades al momento de comprender el orden y jerarquía de las interfaces.

\subsubsection{Segunda iteración}

- Rompecabezas. El usuario (alumno del CAM) utilizó un prototipo de alta fidelidad, de material de acrílico con una imagen diferente a la primera iteración y además interactivo. De esta forma se determinó que al usuario se le dificultaba identificar el lado correcto de la pieza, por ser transparente. También se reconoció que al usuario le gustaba distinguir la forma de las piezas con los dedos. Las tareas que el usuario debía de cumplir fueron: 1) identificar correctamente los dos lados de las piezas, 2) reconocer la forma y el lugar donde se ubica cada pieza y 3) armar el rompecabezas de manera correcta. Se realizó una evaluación diagnóstica en cada tarea para identificar problemas de usabilidad, así como dificultades con el prototipo. En esta iteración se observaron problemas en el diseño del rompecabezas ya que el usuario no logró concluir las tareas de manera correcta o en un tiempo menor.
- Aplicación móvil. Mediante las técnicas de Style Guides y Card Sorting, se eligieron nuevos colores que favorecieran el aspecto visual de la aplicación, se cambiaron algunos iconos para que ayudaran a identificar cada parte del sistema y se hizo un reordenamiento de la jerarquía y contenido de algunas de las interfaces. A través de la técnica de Wizard of $\mathrm{Oz}$, por medio de un prototipo de media fidelidad, se observó que el usuario (tutor) tenía más facilidad al comprender e interactuar con las interfaces.

\subsubsection{Tercera iteración}

- Rompecabezas. Se trabajó en el mejoramiento del diseño del rompecabezas modificando los colores. Por otro lado, debido al gusto del usuario (alumno del CAM) por distinguir las piezas con los dedos, se consideró agregar textura en cada una de las piezas para facilitar la identificación del lado correcto. Las tareas que se realizaron fueron: 1) identificar si el producto es un rompecabezas, 2) identificar el lado correcto de las piezas, 3) reconocer la forma y el lugar donde se ubica cada pieza y 4) armar el rompecabezas de manera correcta. Para la evaluación final, se observaron menos problemas que los anteriores notándose la facilidad por parte del usuario para identificar las piezas, ayudándole los colores, la forma y la textura. Como resultado final el usuario logró interactuar con el rompecabezas en un tiempo récord menor a los anteriores y con una experiencia de uso satisfactoria.

- Aplicación móvil. Se utilizó un prototipo de media fidelidad, a la cual se le agregaron más colores para facilitar el reconocimiento y se pueda retener la atención del usuario (tutor). Así mismo, se cambiaron y agregaron más imágenes para facilitar la comprensión de las funciones disponibles. Lo anterior permitió al usuario identificar fácilmente las funciones, teniendo un manejo más fluido y una buena experiencia de uso.

\subsubsection{Solución Propuesta}

Gracias a los resultados obtenidos con la evaluación en las distintas iteraciones se llegó a la propuesta que se mencionó en la cuarta iteración de la etapa de diseño (Figura 3).
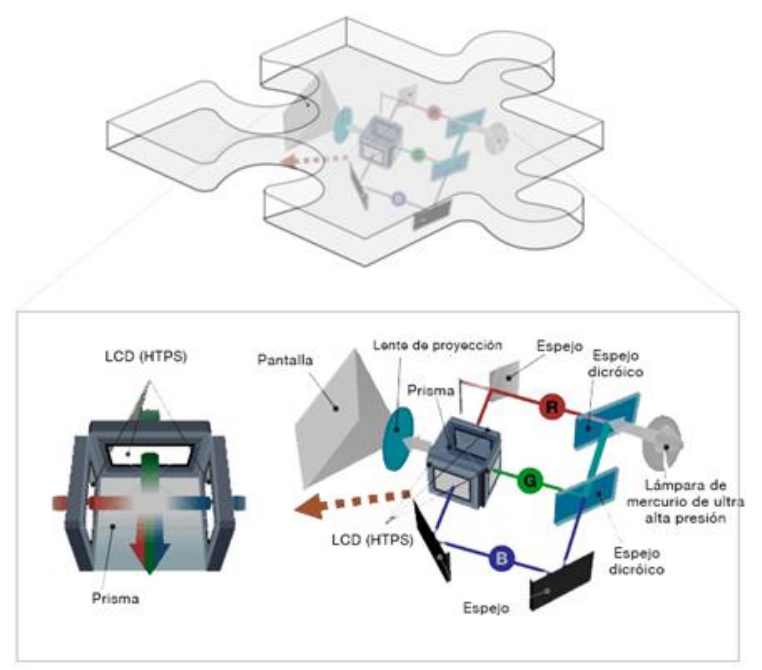

\section{Figura 3. Proyector implementado en una pieza del rompecabezas}

Cabe mencionar, que es una propuesta teórica, ya que por el momento la implementación a un prototipo real nos resulta difícil 
por ser una aplicación tecnológica novedosa. Sin embargo se espera que en un futuro Kandii Puzzle sea accesible a instituciones que atienden a pacientes con discapacidad intelectual moderada.

\section{Impacto social}

Kandii Puzzle es la implementación de la tecnología en la educación como una herramienta de apoyo, ya que no pretende sustituir al tutor, sino que se desea ayudarlo de manera que cuente con más elementos (visuales y auditivos), para enriquecer el proceso de estimulación y enseñanza.

Kandii Puzzle fomenta la inclusión del usuario, beneficiando la diversidad de ideas, gustos, intereses y sobretodo las necesidades y habilidades adaptativas sociales, ya que podría usarse de manera individual o en conjunto.

Este producto no busca ser distribuido de momento (por su costo) de manera individual, se considera en una primera etapa, su distribución en instituciones como el CAM, pudiendo ser compartido con un mayor número de usuarios.

\section{Conclusiones}

El juego es una actividad esencial en el ser humano, ya que forma parte de su desarrollo cognitivo, físico y social [10]. Mediante Kandii Puzzle otorgamos una herramienta tecnológica como una forma de juego de manera que, mientras el usuario se divierte, obtendrá una estimulación temprana de sus características sensoriales, motrices y cognitivas que muy posiblemente pueda beneficiar su calidad de vida en un futuro.

Kandii Puzzle resultó efectivo porque el usuario logró completar las tareas, mejorando su tiempo de realización de 15 a 9 minutos. También observamos que el producto logra una satisfacción al usuario al cumplir con los objetivos del juego.

Lo anterior gracias a la metodología UCD-e, donde no solo se buscan los elementos principales de la usabilidad, sino que también se enfoca en transmitir emociones por medio de la Interacción Humano-Computadora.

\section{Referencias}

[1] Allen Leigh, Betania, Estudio sobre discriminación y discapacidad mental e intelectual, Dirección General Adjunta de Estudios, Legislación y Políticas Públicas. Diciembre 2009. Disponible en:

http://www.conapred.org.mx/documentos_cedoc/E062009.pdf Documento de Trabajo No. E-06-2009.

[2] Carpio Brenes, María de los Ángeles, La tecnología asistiva como disciplina para la atención pedagógica de personas con discapacidad intelectual. Revista Electrónica "Actualidades Investigativas en Educación" [en línea] 2012, 12 (Mayo-
Agosto): [Fecha de consulta: 4 de junio de 2018] Disponible en: http://www.redalyc.org/articulo.oa?id=44723437018 ISSN

[3] Consejo Consultivo de UNICEF México, Vigía de los derechos de la niñez mexicana, UNICEF. Diciembre 2005. Disponible en https://www.unicef.org/mexico/spanish/mx_resources_vigia_ II.pdf

[4] García Alonso, Isabel, Concepto actual de discapacidad intelectual. Psychosocial Intervention [en línea] 2005, 14 (Sin mes): [Fecha de consulta: 4 de junio de 2018] Disponible en: http://www.redalyc.org:9081/articulo.oa?id=179817547002 ISSN 1132-0559

[5] Harper Richard; Rodden Tom; Rogers Yvonne; Sellen Abigail, BEING HUMAN: Human computer interaction in the year 2020, Microsoft Research Ltd., 2008. ISBN: 978-09554761-1-2

[6] INEGI, La discapacidad en México, datos al 2014, Instituto Nacional de Estadística y Geografía, 2016. Disponible en: http://conadis.gob.mx/gob.mx/transparencia/transparencia_fo calizada/La_Discapacidad_en_Mexico_datos_2014.pdf

[7] Jasarevic, Tarik, Los niños con discapacidad son víctimas de la violencia con más frecuencia, Organización Mundial de la Salud, 12 de Julio de 2012. Disponible en: http://www.who.int/mediacentre/news/notes/2012/child_disa bilities_violence_20120712/es/

[8] Ke X, Liu J. Discapacidad intelectual (Irarrázaval M, Martin A, Prieto-Tagle F, Fuertes O. trad.). En Rey JM (ed), Manual de Salud Mental Infantil y Adolescente de la IACAPAP. Ginebra: Asociación Internacional de Psiquiatría del Niño y el Adolescente y Profesiones Afines 2017.

[9] Mora Tere, 5 beneficios que tiene para la mente el armar puzzles, s/n, www.Vix.com, Disponible en: https://www.vix.com/es/imj/familia/6598/5-beneficios-quetiene-para-la-mente-el-armar-puzzles

[10] Victoria, Uriba; Uría Alejandra; López Juan José, ¿Los juguetes representan la diversidad de los usuarios? Una relfexión acerca de la responsabilidad social en su diseño. Revista Digital Universitaria UNAM. Julio-Agosto 2017. Disponible en: http://www.revista.unam.mx/vol.18/num6/art45/index.html ISSN: $1607-6079$ 